\title{
Observer-based consensus tracking of multi-agent systems with one-sided Lipschitz nonlinearity
}

\author{
Hongjun Chu, Xiaocheng Liu, Weidong Zhang and Yunze Cai
}

Department of Automation, Shanghai Jiao Tong University, and Key Laboratory of System Control and Information Processing, Ministry of Education of China, Shanghai, 200240, China

\begin{abstract}
This paper investigates the observer-based consensus tracking problem of multiagent systems with one-sided Lipschitz nonlinearity. The agent dynamics considered here covers a broad family of nonlinear systems, and includes the well-known Lipschitz system as a special case. To achieve consensus tracking for such multi-agent systems, two types of observer-based protocols named the continuous protocol and the intermittent protocol are proposed. Furthermore, several multi-step design algorithms are presented to select the observer gains and the controller parameters of the proposed protocols. It is shown that the established sufficient criteria can not only ensure the observer error to approach to zero, but also realize the consensus tracking of multi-agent systems. The obtained results are illustrated by two simulation examples.
\end{abstract}

Keywords: multi-agent systems, one-sided Lipschitz nonlinearity, observer, intermittent protocol.

\section{Introduction}

In the area of cooperative control, consensus is an important and fundamental problem. By designing distributed protocols, the purpose of the consensus for multi-agent systems is to make a group of agents to reach an agreement on certain quantities of interest [1]. It is well-known that the agent dynamics and the interaction topology are two key factors in achieving consensus, and much effort has been made along this line. In most existing results, the agents dynamics are assumed to be first-, second-, or high-order integrators, and general linear systems. Recently, the consensus problems of nonlinear multi-agent systems have been studied in [2-3], where the plant system consists of a linear part and a Lipschitzt nonlinear part. To reduce influence of the nonlinearity, the Lipschitz constant is desired as small as possible. However, this constant is based-region, and often dramatically increases as the operating region is enlarged. To overcome this drawback, the Lipschitz condition was generalized to one-sided Lipschitz one. This extension is motivated by some important applications such as numerical analysis [4], stiff problem [5-6] and birth death problem of a population [7]. For these problems, the one-sided Lipschitz constant is significantly smaller than the classical Lipschitz constant [8]. This makes it much more appropriate for estimating the influence of the nonlinear term. That is, One-sided Lipschitz can dig deeply into the nature of nonlinearity, and explore more information on the nonlinearity term. Hence, it

E-mail : chuhongjun2007@126.com (H. Chu), liuxc12345@gmail.com (X. Liu),wdzhang@sjtu.edu.cn ( W. Zhang). 
possesses inherent advantages in the control and observation theory, and in particular to address the observer design problem [9]. However, the existing investigations on the one-sided Lipschitz are only confined in the single system framework. To the best of our knowledge, until now, no results have been found on the study of consensus tracking for multi-agent systems with one-sided Lipschitz, and this motivates our research.

The relative states between neighboring agents are not always available, while the relative outputs are easy to acquire. Thus, consensus by output feedback is easier to implement than one by state feedback in practice. Due to static output feedback being quite restrictive [10], observer-based (or dynamic) output feedback is an elegant alternative [11]. Existing results on this topic can be found in the literatures. To track an active leader, a novel distributed estimator-based protocol was designed in [12] for first-order agents. Subsequently, this result was extended to the second-order case in [13], and the finite-time tracking control in [14], respectively. For multi-agent systems with general linear dynamics, an observer-based consensus protocol was proposed in [15] by using the relative output information. Besides this full-order observer, the reduced-order observer was further investigated in [16]. A consensus tracking framework, including state feedback and output feedback, was presented for continuous-time multi-agent systems in [17], and the discrete-time counterpart in [18]. It should be pointed out that most of the existing results with respect to observer-type protocols mainly focus on integrator systems or general linear systems. Until now, few works on nonlinear multi-agent systems are found [19]. The traditional observer problem of a single nonlinear system has been studied for decades [8, 9, 20-22], and there are mainly four different approaches to design nonlinear observer [23]. Here, we only consider the case of one-sided Lipschitz agents. More specifically, the systems dynamics can be split into a linear part and a nonlinear part, where the linear part is assumed to be observable from system output, and the nonlinear part is one-sided Lipschitz.

On the other hand, the control inputs are inevitably missing in real control systems due to: 1) some unpredictable external factors that break edges or vertexes, e.g., actuator failure, packet loss; 2) some intentional reasons such as communication cost limitation, and the controllers are purposefully suspended from time to time. The occasionally missing control inputs can make control discontinuous, e.g., intermittent control and impulsive control. Compared with the continuous case, the discontinuous control method is more economical and efficient. Therefore, it has aroused great interest among researchers, since it was proposed in [24]. More recently, the intermittent protocols were proposed in [25] to reach consensus tracking of linear multi-agent systems. This intermittent idea was also adopted in containment control of multi-agent systems with intermittent input saturation [26], and synchronization of complex network via randomly occurring control [27]. However, these results are only restricted in state feedback framework rather than output feedback case.

Motivated by the above discussions, this paper aims to design the observer-based protocols for achieving consensus tracking for multi-agent systems with one-sided Lipschitz nonlinearity. Based on relative outputs between agents, we first design distributed state observers, and then, by using the observed states, construct the continuous protocol and the intermittent protocol. In summary, the main contributions of this paper are threefold: (1) we introduce one-sided Lipschitz condition in [9, 20-22] into multi-agent systems, and then extend the traditional observer design for a single nonlinear system to the observer-based 
protocols design for multi-agent systems with one-sided Lipschitz; (2) Two types of observer-based protocols, namely the continuous protocol and the periodically intermittent protocol are proposed here. Compared with the former, the latter seems more practical and environment-friendly in engineering. The observer leads to dimension expansion of closed-loop systems, together with nonlinear term and intermittent communication network jointly making convergence analysis intractable. The multiple Lyapunov functions based on dwell time approach is used to overcome this difficulty. Compared to existing results, we extend the state feedback protocols in $[2,3]$ to the dynamic output feedback protocols, and extend continuous observer-based protocols in [19] to the intermittent case. (3) Multi-step algorithms are given to design the observer gains and the protocol parameters, which make the corresponding protocols easy to implement. Based on these algorithms, the sufficient criteria for achieving the consensus tracking are established. It should be pointed out that these criteria contain the dwell time information regarding topological graphs. Moreover, compared with the existing results, our results are more general and less conservative.

The remainder of the paper is organized as follows. Section 2 provides some preliminaries and problem formulation. In Section 3, we construct two kinds of observer-based protocols, and analytically prove that they can solve consensus tracking problem for multi-agent systems with one-sided Lipschitz nonlinearity. Two simulation examples are given in Section 4, and finally, Section 5 concludes the whole paper.

\section{Preliminaries and problem formulation}

\subsection{Preliminaries}

${ }^{n}$ denotes the $n$ dimensional real Euclidean space. Let $\square$ be the non-negative integer set. $(\cdot, \cdot)$ is the inner product in $\square^{n}$, i.e., for given $x, y \in \square^{n}$, then $(x, y)=x^{T} y$, where $x^{T}$ is the transpose of $x . \lambda_{\min }(P)$ and $\lambda_{\max }(P)$ denote the minimal and maximum eigenvalues of the symmetric matrix $P$, respectively. In symmetric block matrices, an asterisk ' $*$ ' represents a term induced by symmetry.

This paper considers a group of agents consisting of $N$ followers and one leader. The information exchange among $N$ followers is described by a weighed digraph $\mathrm{G}=(\mathrm{V}, \mathrm{E}, \mathrm{A})$, where a node set is $\mathrm{V}=\left\{v_{1}, v_{2}, \cdots, v_{N}\right\}$ and an edge set is $\mathrm{E} \subseteq \mathrm{V} \times \mathrm{V}$, and an adjacency matrix is $\mathrm{A}=\left[a_{i j}\right] \in \square^{N \times N}$, and $a_{i j}>0 \Leftrightarrow\left(v_{j}, v_{i}\right) \in \mathrm{E}$, and $a_{i j}=0$ otherwise. The edge $\left(v_{j}, v_{i}\right)$ means agent $i$ can receive information from $j$ but not conversely. The Laplacian

matrix of $\mathrm{G}$ is denoted by $\mathrm{L}=\left[l_{i j}\right]$, where $l_{i j}=-a_{i j}, i \neq j$, and $l_{i i}=\sum_{j=1, j \neq i}^{N} a_{i j}$. We define the augmented graph $\tilde{\mathrm{G}}$ consisting of $N$ followers and a leader. The information 
acquisition of the followers to the leader is represented by $\mathrm{D} \square \operatorname{diag}\left\{d_{1}, \cdots, d_{N}\right\}$, where $d_{i}>0$ if the follower $i$ has access to the leader's state, and $d_{i}=0$ otherwise.

Assumption 1. The directed graph $\tilde{\mathrm{G}}$ contains a spanning tree with the root node being the leader.

Denote $\tilde{\mathrm{L}} \square \mathrm{L}+\mathrm{D}$. A useful property is given in the following lemma.

Lemma 1 [28]. If Assumption 1 holds, $\tilde{L}$ is invertible. Moreover, denote $\left(\theta_{1} \cdots, \theta_{N}\right)^{T}=\left(\tilde{\mathrm{L}}^{T}\right)^{-1} \mathbf{1}_{N}$ and $\Theta=\operatorname{diag}\left\{\theta_{1}, \cdots, \theta_{N}\right\}$, then $\Theta$ and $\Theta \tilde{\mathrm{L}^{2}}+\tilde{\mathrm{L}}^{T} \Theta$ are positive definite matrices.

\subsection{Problem formulation}

In this paper, the main purpose is to solve the consensus tracking problem for general linear multi-agent systems with one-sided Lipschitz. Base on the relative outputs between the neighboring agents, we construct state observers for each nonlinear agent, and then design distributed protocols by using the observed states.

Consider a group of $N$ nonlinear agents, the dynamics of agent $i$ is given by

$$
\left\{\begin{array}{l}
\dot{x}_{i}=A x_{i}+D \Phi\left(x_{i}, t\right)+B u_{i}, \\
y_{i}=C x_{i}, \quad i=1, \cdots, N .
\end{array}\right.
$$

where the state $x_{i} \in R^{n}$, control input $u_{i} \in R^{p}$, and the measurement output $y_{i} \in R^{q}$.

$A, B, C, D$ are constant real matrices with appropriate dimensions, and suppose that $(A, B, C)$ is controllable and observable. The function $\Phi(\cdot, \cdot): R^{n} \times R \rightarrow R^{n}$ is a continuous vector-valued function denoting the intrinsic nonlinearity, which satisfies the following assumption.

Assumption 2. 1). The nonlinear function $\Phi$ is said to be one-sided Lipschitz, if there exists $\rho \in R$ such that $\forall x, y \in D_{1} \subseteq R^{n}$

$$
(\Phi(x, t)-\Phi(y, t), x-y) \leq \rho\|x-y\|^{2}
$$

where the smallest $\rho \in R$ is called one-side Lipschitz constant, which depends on local region $D_{1}$ and dynamics $\Phi$. If the condition (2) is valid everywhere in $R^{n}$, then the function is said to be globally one-side Lipschitz.

2).The nonlinear function $\Phi$ is said to be quadratic inner-bounded if there exist 
$\mu, v \in R$ such that $\forall x, y \in D_{2} \subseteq R^{n}$

$$
\|\Phi(x, t)-\Phi(y, t)\|^{2} \leq \mu\|x-y\|^{2}+v(\Phi(x, t)-\Phi(y, t), x-y) .
$$

Noticeably, the constants $\rho, \mu$ and $v$ can be positive, negative or zero, while the well-known Lipschitz constant must be positive. Additionally, if the function $\Phi\left(x_{i}, t\right)$ is Lipschitz, then it is also both one-sided Lipschitz (e.g. with $\rho>0$ ) and quadratically inner-bounded (e.g. with $\mu>0$ and $v=0$ ), but not vice versa [20].

Now we give an example that satisfies one sided Lipschitz condition but not Lipschitz.

Consider Newton's law of universal gravitation $F=\frac{G m_{1} m_{2}}{r^{2}}$, i.e., $r=\frac{\sqrt{G m_{1} m_{2}}}{\sqrt{F}}$. For given $m_{1}$ and $m_{2}$, denote $a \square \sqrt{G m_{1} m_{2}}, f(x) \square r$ and $x \square F$, radius $f(x)$ is the nonlinear function in force $x$, which can be expressed by $f(x)=\frac{a}{\sqrt{x}},(a>0)$. For any $x_{1}, x_{2} \in(0, b]$, and every $M>0$, when $x_{1}$ and $x_{2}$ both approach to 0 , we have $\left\|f\left(x_{1}\right)-f\left(x_{2}\right)\right\|=\left\|\frac{a}{\sqrt{x_{1}}}-\frac{a}{\sqrt{x_{2}}}\right\|=\frac{a\left\|x_{1}-x_{2}\right\|}{\sqrt{x_{1} x_{2}}\left(\sqrt{x_{1}}+\sqrt{x_{2}}\right)} \geq M\left\|x_{1}-x_{2}\right\|$, which implies that $f(x)$ is not Lipschitz on any interval $(0, b]$. Conversely, we have $\left(f\left(x_{1}\right)-f\left(x_{2}\right), x_{1}-x_{2}\right)=\left(\frac{a}{\sqrt{x_{1}}}-\frac{a}{\sqrt{x_{2}}}\right)\left(x_{1}-x_{2}\right)=\frac{-a\left(x_{1}-x_{2}\right)^{2}}{\sqrt{x_{1} x_{2}}\left(\sqrt{x_{1}}+\sqrt{x_{2}}\right)} \leq-\frac{a}{2 b \sqrt{b}}\left(x_{1}-x_{2}\right)^{2}$ thus, $f(x)$ is locally one sided Lipschitz with one sided Lipschitz constant $-\frac{a}{2 b \sqrt{b}}$. In addition, $f(x)$ is not quadratic inner-bounded, but some systems can simultaneously satisfy conditions (2) and (3), e.g., the famous supercritical Hopf bifurcation system, which is elaborated in Example 2 of Section 4.

Remark 1. The nonlinear term of Example 1 in Section 4 satisfies $\left(x_{1}-x_{2}\right)\left(f\left(x_{1}\right)-f\left(x_{2}\right)\right)<0$ with $x_{1}, x_{2}>0$ and $x_{1} \neq x_{2}$, which shows that the one-sided Lipschitz condition is different from the incrementally passive or slope-restricted nonlinearities addressed by [29 ].QUAD condition is proposed in [30-31] and it can be viewed as generalization of one-sided Lipschitz condition, so the results obtained in this paper can be easily extended to the case of QUAD condition. 
In many practical cases, it is desired that the agents' states can approach the time-varying reference state $s(t)$, which can be generated by the virtue leader as follows [32].

$$
\dot{s}=A s+D \Phi(s, t), s(0)=s_{0} .
$$

Our control objective is to design the consensus protocols such that each agent in systems (1) can be synchronized to the trajectory (4). In the following section, two kinds of observer-based protocols, including the continuous protocol and the intermittent protocol, are proposed to reach the consensus tracking for nonlinear multi-agent systems.

\section{Main results}

In this section, the main theoretical results of this paper are given and analytically proven.

\subsection{Consensus with the continuous observer-type protocol}

To begin with, the following observer is proposed for systems (1)

$$
\left\{\begin{aligned}
\dot{\hat{x}}_{i}= & A \hat{x}_{i}+D \Phi\left(\hat{x}_{i}, t\right)+B u_{i}+\beta d_{i} F\left(y_{i}-\hat{y}_{i}\right) \\
& +\beta F\left(\sum_{j=1}^{N} a_{i j}\left(\hat{y}_{j}-\hat{y}_{i}\right)-\sum_{j=1}^{N} a_{i j}\left(y_{j}-y_{i}\right)\right), \\
\hat{y}_{i}= & C \hat{x}_{i}, \quad i=1, \cdots, N .
\end{aligned}\right.
$$

where $\hat{x}_{i}$ is the estimation of $x_{i}$, and $\hat{y}_{i}$ is the output of the observer systems. $\beta$ and $F$ are a coupling strength and an observer gain matrix to be determined, respectively. To reduce the control cost, the reference state $s(t)$ is available to only a small fraction of followers, thus the following protocol is given on the basis of the observed states.

$$
u_{i}=-d_{i} \alpha K\left(\hat{x}_{i}-s\right)+\alpha K \sum_{j=1}^{N} a_{i j}\left(\hat{x}_{j}-\hat{x}_{i}\right)
$$

where $\alpha$ and $K$ are a coupling strength and a controller gain matrix to be designed, respectively. $d_{i}>0$ if the agent $i$ acquires the state of the leader, otherwise $d_{i}=0$.

Under the protocol (6), the dynamics concerned with the state and its estimation of systems (1) are given as follows:

$$
\left\{\begin{aligned}
\dot{x}_{i}= & A x_{i}+D \Phi\left(x_{i}, t\right)-d_{i} \alpha B K\left(\hat{x}_{i}-s\right)+\alpha B K \sum_{j=1}^{N} a_{i j}\left(\hat{x}_{j}-\hat{x}_{i}\right), \\
\dot{\hat{x}}_{i}= & A \hat{x}_{i}+D \Phi\left(\hat{x}_{i}, t\right)-d_{i} \alpha B K\left(\hat{x}_{i}-s\right)+\alpha B K \sum_{j=1}^{N} a_{i j}\left(\hat{x}_{j}-\hat{x}_{i}\right) \\
& +\beta d_{i} F C\left(x_{i}-\hat{x}_{i}\right)-\beta F C\left(\sum_{j=1}^{N} a_{i j}\left(x_{j}-\hat{x}_{j}\right)-\sum_{j=1}^{N} a_{i j}\left(x_{i}-\hat{x}_{i}\right)\right) .
\end{aligned}\right.
$$

The estimation errors and the tracking errors are respectively defined as:

$$
e_{1 i}=x_{i}-\hat{x}_{i} \text { and } e_{2 i}=x_{i}-s, i=1, \cdots, N .
$$

It follows from (1) and (7) that 


$$
\left\{\begin{array}{l}
\dot{e}_{1 i}=A e_{1 i}+D \tilde{\Phi}_{1 i}-\beta d_{i} F C e_{1 i}+\beta F C \sum_{j=1}^{N} a_{i j}\left(e_{1 j}-e_{1 i}\right) \\
\dot{e}_{2 i}=A e_{2 i}+D \tilde{\Phi}_{2 i}-\alpha d_{i} B K\left(e_{2 i}-e_{1 i}\right)+\alpha B K \sum_{j=1}^{N} a_{i j}\left(e_{2 j}-e_{2 i}-e_{1 j}+e_{1 i}\right) .
\end{array}\right.
$$

where $\quad \tilde{\Phi}_{1 i} \square \Phi\left(x_{i}, t\right)-\Phi\left(\hat{x}_{i}, t\right) \quad$ and $\quad \tilde{\Phi}_{2 i} \square \Phi\left(e_{2 i}+s, t\right)-\Phi(s, t) \quad . \quad$ By letting $e_{1}=\left[e_{11}^{T}, e_{12}^{T}, \cdots, e_{1 N}^{T}\right]^{T}$ and $e_{2}=\left[e_{21}^{T}, e_{22}^{T}, \cdots, e_{2 N}^{T}\right]^{T}$, then the error system (8) can be written as the following compact form:

$$
\left\{\begin{array}{l}
\dot{e}_{1}=(I \otimes A) e_{1}-\beta \tilde{\mathrm{L}} \otimes F C e_{1}+(I \otimes D) \tilde{\Phi}_{1}, \\
\dot{e}_{2}=(I \otimes A) e_{2}-\alpha \tilde{\mathrm{L}} \otimes B K e_{2}+\alpha \tilde{\mathrm{L}} \otimes B K e_{1}+(I \otimes D) \tilde{\Phi}_{2} .
\end{array}\right.
$$

where $\tilde{\mathrm{L}}$ is defined above in Lemma1, $\tilde{\Phi}_{1}=\left[\tilde{\Phi}_{11}^{T}, \tilde{\Phi}_{12}^{T}, \cdots, \tilde{\Phi}_{1 N}^{T}\right]^{T}$, and $\tilde{\Phi}_{2}=\left[\tilde{\Phi}_{21}^{T}, \tilde{\Phi}_{22}^{T}, \cdots, \tilde{\Phi}_{2 N}^{T}\right]^{T}$.

Before moving forward, a design procedure is given for selecting observer and protocol parameters.

Algorithm 1. Under Assumptions 1 and 2, the observer (5) and the controller (6) can be designed as follows:

1. Solve the following linear matrix inequality (LMI):

$$
\Pi=\left(\begin{array}{cc}
P A+A^{T} P-\sigma C^{T} C+\left(\varepsilon_{1} \rho+\varepsilon_{2} \mu\right) I & P D+\frac{\left(\varepsilon_{2} v-\varepsilon_{1}\right) I}{2} \\
* & -\varepsilon_{2} I
\end{array}\right)<0
$$

to get a matrix $P>0$ and three positive scalars $\varepsilon_{1}, \varepsilon_{2}$ and $\sigma$. Then, choose the observer gain matrix $F=P^{-1} C^{T}$ and the coupling strength $\beta \geq \frac{\sigma \theta_{\max }}{\lambda_{1}}$, where $\lambda_{1} \square \lambda_{\text {min }}\left(\Theta \tilde{\mathrm{L}}+\tilde{\mathrm{L}}^{T} \Theta\right)$ and $\theta_{\max } \square \max _{1 \leq j \leq N}\left\{\theta_{j}\right\}$.

2. For arbitrarily given positive scalars $\varepsilon_{3}, \varepsilon_{4}$, solve the following LMI:

$$
\left(\begin{array}{ccc}
A Q+Q A^{T}-\kappa B B^{T} & D+\frac{\left(\varepsilon_{4} v-\varepsilon_{3}\right) Q}{2} & \left(\varepsilon_{3} \rho+\varepsilon_{4} \mu\right) Q \\
* & -\varepsilon_{4} I & 0 \\
* & * & -\left(\varepsilon_{3} \rho+\varepsilon_{4} \mu\right) I
\end{array}\right)<0
$$

to get a matrix $Q>0$ and a positive scalar $\kappa$. Then, select the controller gain matrix $K=B^{T} Q^{-1}$ and the coupling strength $\alpha \geq \frac{\kappa \theta_{\max }}{\lambda_{1}}$. 
Theorem 1. Let Assumptions 1 and 2 hold. If the LMIs (10) and (11) have feasible solutions, the consensus tracking of systems (1) and (4) can be realized under the observer (5) and the controller (6) constructed by Algorithm 1.

Proof. Consider the following Lyapunov function candidate

$$
V(t)=V_{1}(t)+V_{2}(t)=e_{1}^{T}(\Theta \otimes P) e_{1}+\varepsilon e_{2}^{T}\left(\Theta \otimes Q^{-1}\right) e_{2} .
$$

where $\Theta$ is defined as in Lemma 1, and $P, Q$ are positive define solutions of the LMIs $(10-11) . \quad \varepsilon$ is a small positive constant.

By using facts that $F=P^{-1} C^{T}$ and $\sigma \leq \frac{\beta \lambda_{1}}{\theta_{\max }}$, the derivative of $V_{1}(t)$ along the trajectories of (9) gives

$$
\begin{aligned}
\dot{V}_{1}(t) & =2 e_{1}^{T}(\Theta \otimes P)\left((I \otimes A) e_{1}-\beta \tilde{\mathrm{L}} \otimes F C e_{1}+(I \otimes D) \tilde{\Phi}_{1}\right) \\
& =e_{1}^{T}\left[\Theta \otimes\left(P A+A^{T} P\right)-\beta\left(\Theta \tilde{\mathrm{L}}+\tilde{\mathrm{L}}^{T} \Theta\right) \otimes C^{T} C\right] e_{1}+2 e_{1}^{T}(\Theta \otimes P D) \tilde{\Phi}_{1} \\
& \leq e_{1}^{T}\left[\Theta \otimes\left(P A+A^{T} P-\sigma C^{T} C\right)\right] e_{1}+2 e_{1}^{T}(\Theta \otimes P D) \tilde{\Phi}_{1} \\
& =\sum_{i=1}^{N} \theta_{i}\left(\begin{array}{c}
e_{1 i} \\
\tilde{\Phi}_{1 i}
\end{array}\right)^{T}\left(\begin{array}{cc}
P A+A^{T} P-\sigma C^{T} C & P D \\
* & 0
\end{array}\right)\left(\begin{array}{c}
e_{1 i} \\
\tilde{\Phi}_{1 i}
\end{array}\right) .
\end{aligned}
$$

From (2), we get $\rho e_{1 i}^{T} e_{1 i}-\tilde{\Phi}_{1 i}^{T} e_{1 i} \geq 0$, or equivalently,

$$
\varepsilon_{1} \sum_{i=1}^{N} \theta_{i}\left(\begin{array}{c}
e_{1 i} \\
\tilde{\Phi}_{1 i}
\end{array}\right)^{T}\left(\begin{array}{cc}
\rho I & -\frac{I}{2} \\
* & 0
\end{array}\right)\left(\begin{array}{c}
e_{1 i} \\
\tilde{\Phi}_{1 i}
\end{array}\right) \geq 0
$$

holds for any positive scalar $\varepsilon_{1}$. Similarly, from (3), we have

$$
\varepsilon_{2} \sum_{i=1}^{N} \theta_{i}\left(\begin{array}{c}
e_{1 i} \\
\tilde{\Phi}_{1 i}
\end{array}\right)^{T}\left(\begin{array}{cc}
\mu I & \frac{v I}{2} \\
* & -I
\end{array}\right)\left(\begin{array}{c}
e_{1 i} \\
\tilde{\Phi}_{1 i}
\end{array}\right) \geq 0
$$

where $\varepsilon_{2}$ is an arbitrary positive scalar. Then, adding the left-hand side terms of (14) and (15) to the right-hand side of (13) yields

$$
\begin{aligned}
& \dot{V}_{1}(t) \leq \sum_{i=1}^{N} \theta_{i}\left(\begin{array}{c}
e_{1 i} \\
\tilde{\Phi}_{1 i}
\end{array}\right)^{T}\left(\begin{array}{cc}
\Pi_{11} & \Pi_{12} \\
* & \Pi_{22}
\end{array}\right)\left(\begin{array}{c}
e_{1 i} \\
\tilde{\Phi}_{1 i}
\end{array}\right)^{T} \\
& =\underbrace{\left(\begin{array}{c}
e_{1} \\
\tilde{\Phi}_{1}
\end{array}\right)^{T}}_{\xi_{1}^{T}} \underbrace{\left.\begin{array}{cc}
\Theta \otimes \Pi_{11} & \Theta \otimes \Pi_{12} \\
* & \Theta \otimes \Pi_{22}
\end{array}\right)}_{\Omega_{11}} \underbrace{\left(\begin{array}{c}
e_{1} \\
\tilde{\Phi}_{1}
\end{array}\right)}_{\xi_{1}} \\
& \square \xi_{1}^{T} \Omega_{11} \xi_{1}
\end{aligned}
$$


where $\left(\begin{array}{cc}\Pi_{11} & \Pi_{12} \\ * & \Pi_{22}\end{array}\right) \square \Pi$ is defined in (10). Obviously, $\Pi<0$ means $\Omega_{11}<0$. It is easy to see that $\lim _{t \rightarrow \infty} e_{1}(t)=0$ under the condition $\Pi<0$, which indicates that the estimation error system is asymptotically stable. Then, the observed states of each agent can be used to construct the distributed protocol.

On the other hand, by using facts that $K=B^{T} Q^{-1}$ and $\kappa \leq \frac{\alpha \lambda_{1}}{\theta_{\max }}$, the derivative of

$V_{2}(t)$ along the trajectories of (9) gives

$$
\begin{aligned}
& \dot{V}_{2}(t)=2 \varepsilon e_{2}^{T}\left(\Theta \otimes Q^{-1}\right)\left((I \otimes A) e_{2}-\alpha \tilde{\mathrm{L}} \otimes B K e_{2}+\alpha \tilde{\mathrm{L}} \otimes B K e_{1}+(I \otimes D) \tilde{\Phi}_{2}\right) \\
& \leq \varepsilon e_{2}^{T}\left(\Theta \otimes\left(Q^{-1} A+A^{T} Q^{-1}-\kappa Q^{-1} B B^{T} Q^{-1}\right)\right) e_{2}+2 \varepsilon e_{2}^{T}\left(\Theta \otimes Q^{-1} D\right) \tilde{\Phi}_{2} \\
& +2 \varepsilon \alpha e_{2}^{T} \Theta \tilde{L} \otimes Q^{-1} B K e_{1} \\
& =\varepsilon \sum_{i=1}^{N} \theta_{i}\left(\begin{array}{c}
e_{2 i} \\
\tilde{\Phi}_{2 i}
\end{array}\right)^{T}\left(\begin{array}{cc}
Q^{-1} A+A^{T} Q^{-1}-\kappa Q^{-1} B B^{T} Q^{-1} & Q^{-1} D \\
* & 0
\end{array}\right)\left(\begin{array}{c}
e_{2 i} \\
\tilde{\Phi}_{2 i}
\end{array}\right) \\
& +2 \varepsilon \alpha e_{2}^{T} \Theta \tilde{\mathrm{L}} \otimes Q^{-1} B B^{T} Q^{-1} e_{1}
\end{aligned}
$$

From (2) and (3), we get

$\varepsilon_{3} \varepsilon \sum_{i=1}^{N} \theta_{i}\left(\begin{array}{c}e_{2 i} \\ \tilde{\Phi}_{2 i}\end{array}\right)^{T}\left(\begin{array}{cc}\rho I & -\frac{I}{2} \\ * & 0\end{array}\right)\left(\begin{array}{c}e_{2 i} \\ \tilde{\Phi}_{2 i}\end{array}\right) \geq 0 \quad$ and $\quad \varepsilon_{4} \varepsilon \sum_{i=1}^{N} \theta_{i}\left(\begin{array}{c}e_{2 i} \\ \tilde{\Phi}_{2 i}\end{array}\right)^{T}\left(\begin{array}{cc}\mu I & \frac{v I}{2} \\ * & -I\end{array}\right)\left(\begin{array}{c}e_{2 i} \\ \tilde{\Phi}_{2 i}\end{array}\right) \geq 0$

where $\varepsilon_{3}$ and $\varepsilon_{4}$ are arbitrary positive scalars. From (17) and (18), one gets that

$$
\begin{aligned}
& \dot{V}_{2}(t)<\varepsilon \sum_{i=1}^{N} \theta_{i}\left(\begin{array}{c}
e_{2 i} \\
\tilde{\Phi}_{2 i}
\end{array}\right)^{T}\left(\begin{array}{cc}
\Sigma_{11} & \Sigma_{12} \\
* & \Sigma_{22}
\end{array}\right)\left(\begin{array}{c}
e_{2 i} \\
\tilde{\Phi}_{2 i}
\end{array}\right)^{T}+2 \varepsilon \alpha e_{2}^{T} \Theta \tilde{L} \otimes Q^{-1} B B^{T} Q^{-1} e_{1} \\
& =\varepsilon \underbrace{\left(\begin{array}{c}
e_{2} \\
\tilde{\Phi}_{2}
\end{array}\right)^{T}}_{\xi_{2}^{T}} \underbrace{\left(\begin{array}{cc}
\Theta \otimes \Sigma_{11} & \Theta \otimes \Sigma_{12} \\
* & \Theta \otimes \Sigma_{22}
\end{array}\right)}_{\Omega_{22}} \underbrace{\left(\begin{array}{c}
e_{2} \\
\tilde{\Phi}_{2}
\end{array}\right)}_{\xi_{2}}+2 \varepsilon \underbrace{\left(\begin{array}{c}
e_{1} \\
\tilde{\Phi}_{1}
\end{array}\right)^{T}}_{\xi_{1}^{T}} \underbrace{\left(\begin{array}{cc}
\alpha \tilde{L}^{T} \Theta \otimes Q^{-1} B B^{T} Q^{-1} & 0 \\
0 & 0
\end{array}\right)}_{\Omega_{12}} \underbrace{\left(\begin{array}{c}
e_{2} \\
\tilde{\Phi}_{2}
\end{array}\right)}_{\xi_{2}} \\
& \square \varepsilon \xi_{2}^{T} \Omega_{22} \xi_{2}+2 \varepsilon \xi_{1}^{T} \Omega_{12} \xi_{2}
\end{aligned}
$$

where $\left(\Sigma_{i j}\right)_{2 \times 2}=\left(\begin{array}{cc}Q^{-1} A+A^{T} Q^{-1}-\kappa Q^{-1} B B^{T} Q^{-1}+\left(\varepsilon_{3} \rho+\varepsilon_{4} \mu\right) I & Q^{-1} D+\frac{\left(\varepsilon_{4} v-\varepsilon_{3}\right) I}{2} \\ * & -\varepsilon_{4} I\end{array}\right)$.

By applying Schur complement Lemma and the congruent transformation in matrix to the left hand of (11), we have $\left(\Sigma_{i j}\right)_{2 \times 2}<0$, i.e., $\Omega_{22}<0$. 
From (16) and (19), it can be obtained that

$$
\dot{V}(t) \leq\left(\begin{array}{l}
\xi_{1} \\
\xi_{2}
\end{array}\right)^{T}\left(\begin{array}{cc}
\Omega_{11} & \propto \Omega_{12} \\
* & \delta \Omega_{22}
\end{array}\right)\left(\begin{array}{l}
\xi_{1} \\
\xi_{2}
\end{array}\right) \square \xi^{T} \Omega \xi .
$$

Due to the facts that $\Omega_{11}<0$ and $\Omega_{22}<0$, we can select the small enough positive constant $\varepsilon$ such that $\Omega_{11}-\varepsilon \Omega_{12} \Omega_{22}^{-1} \Omega_{12}^{T}<0$ holds, which is Schur equivalent to $\Omega<0$. Therefore, one concludes that $\lim _{t \rightarrow \infty} e_{1}(t)=0$ and $\lim _{t \rightarrow \infty} e_{2}(t)=0$, which means that the error system (9) is asymptotically stable. Thus, the protocol (6) can solve the observer-based consensus tracking problem for systems (1) and (4). This completes the proof.

The observer (5) of each agent need have access to the information of $y_{j}-y_{i}, \hat{y}_{j}-\hat{y}_{i}$ and $y_{i}-\hat{y}_{i}$ through communication network. This observer architecture requires a significant amount of computations and communication traffic, and then is not easy to implement in practice. To overcome this shortcoming, we will propose a simple and efficient observer. Specifically, the input $u_{i}$ and relative output $y_{i}-\hat{y}_{i}$ is only needed to estimate the agents' state. Thus, this observer architecture (20) considerably reduces communication energy consumption.

A local observer is given on the basis of its own information rather than network information with form

$$
\left\{\begin{array}{l}
\dot{\hat{x}}_{i}=A \hat{x}_{i}+D \Phi\left(\hat{x}_{i}, t\right)+B u_{i}+F\left(y_{i}-\hat{y}_{i}\right), \\
\hat{y}_{i}=C \hat{x}_{i}, \quad i=1, \cdots, N
\end{array}\right.
$$

where the variables are the same in (5). The protocol (6) is still adopted here. By similar deduction, the whole error system can be given as follows:

$$
\left\{\begin{array}{l}
\dot{e}_{1}=I \otimes(A-F C) e_{1}+(I \otimes D) \tilde{\Phi}_{1}, \\
\dot{e}_{2}=(I \otimes A) e_{2}-\alpha \tilde{\mathrm{L}} \otimes B K e_{2}+\alpha \tilde{\mathrm{L}} \otimes B K e_{1}+(I \otimes D) \tilde{\Phi}_{2} .
\end{array}\right.
$$

Similar to Theorem 1, we can establish a sufficient condition for achieving consensus tracking of multi-agent systems. Its proof is omitted due to limited space.

Corollary 1. Let Assumptions 1 and 2 hold. If the LMIs (22) and (23) have feasible solutions, the consensus tracking of systems (1) and (4) can be realized under the observer (20) and the controller (6) constructed by the following procedure.

1. Solve the following LMI:

$$
\left(\begin{array}{cc}
P A+A^{T} P-2 C^{T} C+\left(\varepsilon_{1} \rho+\varepsilon_{2} \mu\right) I & P D+\frac{\left(\varepsilon_{2} v-\varepsilon_{1}\right) I}{2} \\
* & -\varepsilon_{2} I
\end{array}\right)<0
$$

to get a matrix $P>0$ and positive scalars $\varepsilon_{1}, \varepsilon_{2}$. Then, choose the observer gain matrix as 
$F=P^{-1} C^{T}$

2.For arbitrarily given positive scalars $\varepsilon_{3}, \varepsilon_{4}$, solve the following LMI:

$$
\left(\begin{array}{ccc}
A Q+Q A^{T}-\kappa B B^{T} & D+\frac{\left(\varepsilon_{4} v-\varepsilon_{3}\right) Q}{2} & \left(\varepsilon_{3} \rho+\varepsilon_{4} \mu\right) Q \\
* & -\varepsilon_{4} I & 0 \\
* & * & -\left(\varepsilon_{3} \rho+\varepsilon_{4} \mu\right) I
\end{array}\right)<0
$$

to get a matrix $Q>0$ and a positive scalar $\kappa$. Then, select the controller gain matrix as $K=B^{T} Q^{-1}$ and the coupling strength $\alpha \geq \frac{\kappa \theta_{\max }}{\lambda_{1}}$, where $\theta_{\max }$ and $\lambda_{1}$ are defined in Algorithm 1.

\subsection{Consensus with the intermittent observer-type protocol}

In the preceding subsection, the consensus tracking problem has been addressed under the assumption that the consensus protocol is continuous, i.e., each agent has to share relative outputs and estimated states with its neighbors all the time. However, the continuous protocol is hard to implement and unrealistic in engineering practice. Now, we will turn our attention to the consensus tracking with the intermittent protocol.

In what follows, we will extend the fixed topology to switching topological graphs. A switching sign $\sigma(t):[0,+\infty) \rightarrow\{1, \cdots, m\}, m \geq 2$ is introduced to describe the evolution

of the underlying graph $\tilde{G}^{\sigma(t)}$. Denote $\left\{\tilde{G}^{1}, \tilde{G}^{2}, \cdots, \tilde{G}^{m}\right\}$ as the set of all possible switching graphs.

Assumption 3. For each $i=1, \cdots, m$, the graph $\tilde{G}^{i}$ contains a directed spanning tree with the leader being the root.

Assume that there exist time intervals $\left[t_{k}, t_{k+1}\right), k \in \square$ with $\bigcap_{k \in]}\left[t_{k}, t_{k+1}\right)=\Phi$ (null set) and $\bigcup_{k \in \llbracket}\left[t_{k}, t_{k+1}\right)=[0, \infty) \quad, \quad$ and then split $\left[t_{k}, t_{k+1}\right)$ into subintervals $\left[t_{k}^{1}, t_{k}^{2}\right), \cdots,\left[t_{k}^{j}, t_{k}^{j+1}\right), \cdots,\left[t_{k}^{h_{k}-1}, t_{k}^{h_{k}}\right)$ with $t_{k}=t_{k}^{1}, t_{k+1}=t_{k}^{h_{k}}$, such that the underlying topology is time-invariant for $t \in\left[t_{k}^{j}, t_{k}^{j+1}\right), j=1, \cdots h_{k}-2$. Due to various factors, control is intermittently actuated, i.e., the control is periodic, and in each period, the time consists of "work time" and "rest time", which is illustrated in Fig. 1. 


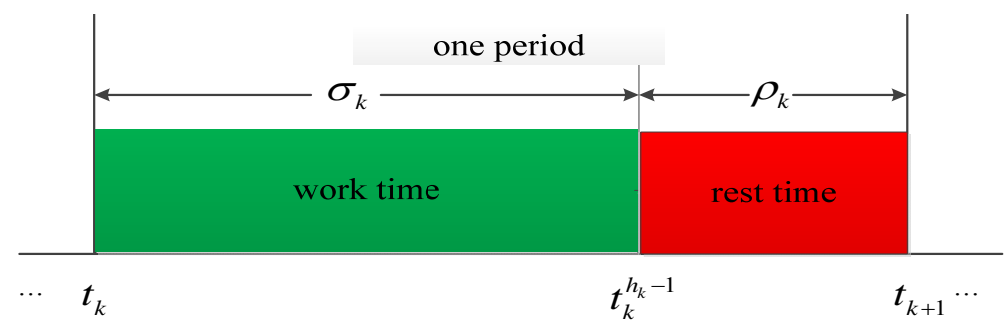

Fig. 1. Sketch of the intermittent control.

Obviously, when $\rho_{k}=0$, the intermittent control degenerates to the traditional continuous

control; when $\sigma_{k}=0$, the intermittent control becomes the impulsive control. Therefore, the intermittent control bridges the gap between the continuous control and the impulsive control.

Due to different reasons, the missing or rebuilding edges inevitably arise in the underlying topology graphs. Once all the edges are simultaneously missing, the null graph (or edgeless graph) appears, while partial edges are missing, the non-null graph arises (here we suppose that each graph is a directed spanning tree). Although event that all the edges are missing (null graph) is a rare event, its existence cannot be ruled out in practice. Thus, in this section, we consider switching graphs between several directed spanning tree graphs and the null graph. When the null graph is actuated, i.e., all the edges are missing, the control signals are automatically set at zero, and hence states and their estimates of each follower evolve according to its own intrinsic dynamics (see 24-b). Moreover, this rest time will last until the work time arrives, and control returns back to normal. Inspired by this, a periodically intermittent observer-type protocol is proposed as follows:

$$
\begin{aligned}
& \left\{\begin{aligned}
\dot{\hat{x}}_{i}= & A \hat{x}_{i}+D \Phi\left(\hat{x}_{i}, t\right)+B u_{i}+\beta d_{i}^{\sigma(t)} F\left(y_{i}-\hat{y}_{i}\right) \\
& +\beta F\left(\sum_{j=1}^{N} a_{i j}^{\sigma(t)}\left(\hat{y}_{j}-\hat{y}_{i}\right)-\sum_{j=1}^{N} a_{i j}^{\sigma(t)}\left(y_{j}-y_{i}\right)\right), \\
\hat{y}_{i}= & C \hat{x}_{i}, \\
u_{i}= & -\alpha K d_{i}^{\sigma(t)}\left(\hat{x}_{i}-s\right)+\alpha K \sum_{j=1}^{N} a_{i j}^{\sigma(t)}\left(\hat{x}_{j}-\hat{x}_{i}\right), t \in\left[t_{k}, t_{k}^{h_{k}-1}\right) .
\end{aligned}\right. \\
& \begin{cases}\dot{\hat{x}}_{i}= & A \hat{x}_{i}+D \Phi\left(\hat{x}_{i}, t\right), \\
u_{i}= & 0, \quad t \in\left[t_{k}^{h_{k}-1}, t_{k+1}\right) .\end{cases}
\end{aligned}
$$

where $a_{i j}^{\sigma(t)}$ is $(i, j)$-th entry of the adjacency matrix $\mathrm{A}^{\sigma(t)}$ associated with $\tilde{\mathrm{G}}^{\sigma(t)}$, and $d_{i}^{\sigma(t)}>0$ if the follower $i$ has access to the leader's state at time $t$, and $d_{i}^{\sigma(t)}=0$ otherwise. The rest of the variables are defined in (5) and (6).

Similar to the above derivation process, the evolution of the estimation error and the consensus error can be described by the following switching systems: 


$$
\begin{aligned}
& \left\{\begin{array}{l}
\dot{e}_{1}=(I \otimes A) e_{1}-\beta \tilde{\mathrm{L}}^{\sigma(t)} \otimes F C e_{1}+(I \otimes D) \tilde{\Phi}_{1}, \\
\dot{e}_{2}=(I \otimes A) e_{2}-\alpha \tilde{\mathrm{L}}^{\sigma(t)} \otimes B K e_{2}+\alpha \tilde{\mathrm{L}}^{\sigma(t)} \otimes B K e_{1}+(I \otimes D) \tilde{\Phi}_{2}, t \in\left[t_{k}, t_{k}^{h_{k}-1}\right)
\end{array}\right. \\
& \left\{\begin{array}{l}
\dot{e}_{1}=(I \otimes A) e_{1}+(I \otimes D) \tilde{\Phi}_{1}, \\
\dot{e}_{2}=(I \otimes A) e_{2}+(I \otimes D) \tilde{\Phi}_{2}, \quad t \in\left[t_{k}^{h_{k}-1}, t_{k+1}\right)
\end{array}\right.
\end{aligned}
$$

where $\tilde{\mathrm{L}}^{\sigma(t)} \square \mathrm{L}^{\sigma(t)}+\mathrm{D}^{\sigma(t)}, \quad \mathrm{D}^{\sigma(t)} \square \operatorname{diag}\left\{d_{1}^{\sigma(t)}, \cdots, d_{N}^{\sigma(t)}\right\}$ and $\mathrm{L}^{\sigma(t)}$ is the Laplacian matrix of $\tilde{G}^{\sigma(t)}$. We can see that the observer-based consensus tracking problem for systems (1) and (4) is transformed into the stability problem of the nonlinear switching systems (25-a, b). Therefore, the switching system theory can be applied to solve this problem.

An algorithm is proposed to select parameters of the intermittent protocol.

Algorithm 2. Under Assumptions 2 and 3, the observer-type protocol (24-a, b) can be designed as follows:

1. Solve the following LMIs: $\left(\tilde{\mathrm{L}}^{i}\right)^{T} \theta^{i}=\mathbf{1}_{N}$ to get vectors $\theta^{i}=\left(\theta_{1}^{i}, \cdots, \theta_{N}^{i}\right)^{T}$ and matrices $\Theta^{i}=\operatorname{diag}\left\{\theta_{1}^{i}, \cdots, \theta_{N}^{i}\right\}$. Moreover $\Theta^{i}>0$ and $\Theta^{i} \tilde{\mathrm{L}}+\tilde{\mathrm{L}}^{T} \Theta^{i}>0, \quad i=1, \cdots, m$. Denote, $\quad r_{1} \square \max _{1 \leq i \leq m, 1 \leq j \leq N}\left\{\theta_{j}^{i}\right\} \quad, \quad r_{2} \square \min _{1 \leq i \leq m, 1 \leq j \leq N}\left\{\theta_{j}^{i}\right\} \quad, \quad r_{0} \square \frac{r_{1}}{r_{2}} \quad$ and $\lambda_{2} \square \min _{1 \leq i \leq m} \lambda_{\min }\left(\Theta^{i} \tilde{L}^{i}+\left(\tilde{L^{i}}\right)^{T} \Theta^{i}\right)$.

2. Solve the following LMIs:

$$
\begin{gathered}
\left(\begin{array}{cc}
P A+A^{T} P-\sigma C^{T} C+\left(\varepsilon_{1} \rho+\varepsilon_{2} \mu\right) I+\delta P & P D+\frac{\left(\varepsilon_{2} v-\varepsilon_{1}\right) I}{2} \\
* & -\varepsilon_{2} I
\end{array}\right)<0 \\
\left(\begin{array}{cc}
P A+A^{T} P+\left(\varepsilon_{1} \rho+\varepsilon_{2} \mu\right) I-\eta P & P D+\frac{\left(\varepsilon_{2} v-\varepsilon_{1}\right) I}{2} \\
* & -\varepsilon_{2} I
\end{array}\right)<0
\end{gathered}
$$

to get a matrix $P>0$ and three positive scalars $\varepsilon_{1}, \varepsilon_{2}$ and $\sigma$. Then, choose the observer gain matrix $F=P^{-1} C^{T}$ and the coupling strength $\beta \geq \frac{\sigma \cdot r_{1}}{\lambda_{2}}$.

3. For arbitrarily given positive scalars $\varepsilon_{3}, \varepsilon_{4}$, solve the following LMIs: 


$$
\begin{gathered}
\left(\begin{array}{ccc}
A Q+Q A^{T}-\kappa B B^{T}+\delta Q & D+\frac{\left(\varepsilon_{4} v-\varepsilon_{3}\right) Q}{2} & \left(\varepsilon_{3} \rho+\varepsilon_{4} \mu\right) Q \\
* & -\varepsilon_{4} I & 0 \\
* & * & -\left(\varepsilon_{3} \rho+\varepsilon_{4} \mu\right) I
\end{array}\right)<0 \\
\left(\begin{array}{ccc}
A Q+Q A^{T}-\eta Q & D+\frac{\left(\varepsilon_{4} v-\varepsilon_{3}\right) Q}{2} & \left(\varepsilon_{3} \rho+\varepsilon_{4} \mu\right) Q \\
* & -\varepsilon_{4} I & 0 \\
* & * & -\left(\varepsilon_{3} \rho+\varepsilon_{4} \mu\right) I
\end{array}\right)<0
\end{gathered}
$$

to get a matrix $Q>0$ and a positive scalar $\kappa$. Then, select the controller gain matrix $K=B^{T} Q^{-1}$ and the coupling strength $\alpha \geq \frac{\kappa \cdot r_{1}}{\lambda_{2}}$.

Theorem 2. Under Assumptions 2 and 3, if the LMIs (26-29) have feasible solutions and inequality (30) also holds, the consensus tracking of systems (1) and (4) can be reached exponentially fast under the observer-type protocol (24-a, b) constructed by Algorithm 2 .

$$
\delta \cdot \sigma_{k}>\eta \cdot \rho_{k}+\left(h_{k}-1\right) \cdot \ln r_{0}
$$

where positive parameters $\delta$ and $\eta$ are determined from (30), and then substituted into the LMIs (26-29). $\sigma_{k} \square t_{k}^{h_{k}-1}-t_{k}, \quad \rho_{k} \square t_{k}^{h_{k}}-t_{k}^{h_{k}-1}, k \in \square$, and $r_{0} \square \frac{r_{1}}{r_{2}}$.

Proof. Construct the following multiple Lyapunov function candidate for switching systems (21-a, b).

$$
V(t)= \begin{cases}e_{1}^{T}\left(\Theta^{i} \otimes P\right) e_{1}+\varepsilon e_{2}^{T}\left(\Theta^{i} \otimes Q^{-1}\right) e_{2}, & t \in\left[t_{k}, t_{k}^{h_{k}-1}\right), \\ e_{1}^{T}(I \otimes P) e_{1}+\varepsilon e_{2}^{T}\left(I \otimes Q^{-1}\right) e_{2}, & t \in\left[t_{k}^{h_{k}-1}, t_{k+1}\right) .\end{cases}
$$

where $\Theta^{i}$ are defined in Algorithm 2, and $P, Q$ are the positive definite solutions of (26-29), $i=1, \cdots, m, k \in \square$. The subsequent proof is very similar to that of Theorem 1 , and the necessary parts are given below.

For $t \in\left[t_{1}, t_{1}^{2}\right)$, we take the time derivative of $V(t)$ along the trajectories of $(25-\mathrm{a})$. Together with inequalities (26) and (28), one has $\dot{V}(t)+\delta V(t) \leq 0$, and further has $V\left(t_{1}^{2-}\right) \leq e^{-\delta\left(t_{1}^{2}-t_{1}\right)} V\left(t_{1}\right)$. On other hand, it follows from (31) that $V\left(t_{1}^{2}\right) \leq r_{0} V\left(t_{1}^{2-}\right)$, where $r_{0}$ is defined in step 1 of Algorithm 2. Thus, one gets $V\left(t_{1}^{2}\right) \leq r_{0} e^{-\delta\left(t_{1}^{2}-t_{1}\right)} V\left(t_{1}\right)$, i.e., 
$V\left(t_{1}^{2}\right) \leq e^{-\delta\left(t_{1}^{2}-t_{1}\right)+\ln r_{0}} V(0)$. By recursion, one can get $V\left(t_{1}^{h_{1}-1}\right) \leq r_{1} e^{-\delta\left(t_{1}^{h_{1}-1}-t_{1}\right)+\left(h_{1}-2\right) \ln \ddot{r}_{0}} V(0)$

For $t \in\left[t_{1}^{h_{1}-1}, t_{2}\right)$, we take the time derivative of $V(t)$ along the trajectories of (24-b), together with (27) and (29), and have that $\dot{V}(t)-\eta V(t) \leq 0$, i.e., $V\left(t_{2}^{-}\right) \leq e^{\eta\left(t_{2}-t_{1}^{\eta_{1}-1}\right)} V\left(t_{1}^{h_{1}-1}\right)$.

Since the systems (25-a, b) switch at $t=t_{2}$, it follows from (31) that

$$
\begin{aligned}
V\left(t_{2}\right) & \leq \frac{1}{r_{2}} V\left(t_{2}^{-}\right) \\
& \leq \frac{r_{1}}{r_{2}} e^{\eta\left(t_{2}-t_{1}^{h_{1}-1}\right)-\delta\left(t_{1}^{h_{1}-1}-t_{1}\right)+\left(h_{1}-2\right) \ln \ddot{r}_{0}} V(0) . \\
\square & e^{-\tau_{1}} V(0)
\end{aligned}
$$

where $\tau \mathrm{k}=\delta \sigma_{1}-\eta \rho_{1}-\left(h_{1}-1\right) \ln r_{0}$. By recursion, one gets that $V\left(t_{k+1}\right) \leq e^{-\sum_{j=1}^{k} \tau_{j}} V(0)$, where $\tau \mathrm{k}=\delta \sigma_{j}-\eta \rho_{j}-\left(h_{j}-1\right) \ln r_{0}$. Moreover, from (30), $\tau \mathrm{k}>0$ hold for all $j=1,2, \cdots, k$ and $k \in \square$.

For any given $t>t_{2}$, there exists an integer $z>2$ such that $t_{z} \leq t<t_{z+1}$, thus time $t$ has three cases.

(a) For the case $t \in\left[t_{z}, t_{z}^{h_{z}-1}\right.$ ), based on the above analysis, one gets

$$
V(t) \leq e^{-\delta\left(t-t_{z}\right)+\left(h_{z}-2\right) / n r_{0}} V\left(t_{z}\right) \leq e^{\left(h_{z}-2\right) / n r_{0}} V\left(t_{z}\right) \leq \mathfrak{J}_{0} e^{-\frac{(z-1) \tilde{\tau} t}{z \omega}} V(0) .
$$

where $\mathfrak{J}_{0}=\left(h_{z}^{\max }-2\right) \ln \vec{r}_{0}, \quad h_{z}^{\max }=\sup _{z \in \square} h_{z}, \quad \tilde{\tau}=\min _{k \in \square} \tau$ k,$\quad \max _{k \in \square}\left(t_{k+1}-t_{k}\right) \leq \omega ，$ $k \in \square$. Since $z>2$, it follows from (28) that

$$
V(t) \leq \mathfrak{J}_{0} e^{-\frac{\tilde{\tau} t}{2 \omega}} V(0) \text {. }
$$

(b) For the case $t=t_{z}^{h_{z}-1}, \quad z>2$, we have

$$
V(t) \leq \frac{\mathfrak{I}_{0}}{r_{2}} e^{-\frac{(z-1) \tilde{\tau} \cdot t}{z \omega_{1}}} V(0) \leq \frac{\mathfrak{I}_{0}}{r_{2}} e^{-\frac{\tilde{\tau} \cdot t}{2 \omega}} V(0)
$$

(c) For the case $t \in\left(t_{z}^{h_{2}-1}, t_{z+1}\right)$, it follows from the fact that $V(t) \leq V\left(t_{z+1}\right)$

$$
V(t) \leq e^{-\sum_{j=1}^{2} \tau_{j} j} V(0) \leq e^{-\frac{\tilde{\tau} \cdot t}{\omega}} V(0)
$$

According to (31) and (33)-(35), we can conclude that $\Upsilon_{1}\left\|\begin{array}{l}e_{1}(t) \\ e_{2}(t)\end{array}\right\|^{2} \leq \Upsilon_{2} e^{-2 \tilde{\tau} t} V(0)$ 
or equivalently $\quad\left\|\begin{array}{l}e_{1}(t) \\ e_{2}(t)\end{array}\right\| \leq \sqrt{\frac{\Upsilon_{2}}{\Upsilon_{1}}} e^{-\tau t t} V(0)$

where $\Upsilon_{1}=\min \left\{r_{1}^{-1} \lambda_{\min }(P), r_{1}^{-1} \lambda_{\min }\left(Q^{-1}\right), \varepsilon \lambda_{\text {min }}(P), \varepsilon \lambda_{\min }\left(Q^{-1}\right)\right\}, \Upsilon_{2}=\max \left\{\mathfrak{I}_{0}, 1, r_{2}^{-1} \mathfrak{I}_{0}\right\}$, and $\vec{\tau}=\frac{\tilde{\tau}}{4 \omega}$. Thus, the consensus tracking for multi-agent systems (1) and (4) can be reached under the observer-type protocol $(24-\mathrm{a}, \mathrm{b})$ constructed by Algorithm 2. This completes the proof.

Remark 2. The most existing results on consensus under time-varying topology are assumed to be arbitrary switching, which is based on the common Lyapunov function (CLF) method [33-35]. However, the arbitrary switching can potentially destroy the stability of a switching system, if the switches happen too frequently [36]. Besides, it is not easy to find, even do not possess a CLF to guarantee the switching system to be stable. Hence, the slow switching topology is considered here. The dwell time of each subsystem needs to satisfy inequality (30) and the multiple Lyapunov function is artfully constructed in (31). From the proof of Theorem 2, we can know that the energy function is exponentially decreasing during the former sub-period, and is exponentially increasing during the latter sub-period. However, from the whole period point of view, the energy function is decreasing in the average sense. Thus, the trajectories of the switching system $(25-\mathrm{a}, \mathrm{b})$ can exponentially converge to zero.

Remark 3. The switching error system $(25-\mathrm{a}, \mathrm{b})$ consists of stable modes and unstable modes. The stable modes are caused by the topologies with several directed spanning trees, while the unstable modes are induced by the null-graph (i.e., all the edges are simultaneously missing).

As reported in [37], the stability of systems $(25-\mathrm{a}, \mathrm{b})$ can be achieved if the total activation time of stable modes is long enough and the decay rates of stable modes are large enough. In this case, the negative effect of the unstable modes can be compensated, and this point can be observed from inequality (30). In this paper, topology structures and weights are assumed to be given prior. Under this assumption, observer-type protocols are designed to realize consensus tracking. Our work is different from the work in Yang et al. [38], where switching topologies and weights were designed to achieve target aggregation for a class of nonlinear multi-agent systems.

Remark 4. In (24-b), $u_{i}=0, i=1, \cdots, N$ means that the control inputs are completely missing. When the missing control inputs are nonexistent, i.e., $\rho_{k}=0$ for all $k \in \square$, and all the switching graphs are also the same, i.e., $\tilde{G}^{1}=\tilde{G}^{2}=\cdots=\tilde{G}^{m}$, the observer-type protocol (24-a, b) reduces to (5-6), and Theorem 2 also degenerates to Theorem 1.

Theorems 1 and 2 provide two observer-type protocols for achieving the consensus tracking for multi-agent systems with one-sided Lipschitz nonlinearity. Since the one-sided Lipschitz condition includes the usual Lipschitz counterpart as a special case, we can derive the following Corollaries 1 and 2 from Theorems 1 and 2, respectively.

Assumption 4. The non-linear function $\Phi$ is said to be locally Lipschitz, if there exists a constant $l>0$ such that $\forall x, y \in D_{3} \subseteq R^{n}$ 


$$
\|\Phi(x, t)-\Phi(y, t)\| \leq l\|x-y\| .
$$

The smallest constant $l>0$ satisfying (37) is known as the "Lipchitz constant".

Corollary 2. Let Assumptions 1 and 4 hold. If the LMIs (38) and (39) have feasible solutions, the consensus tracking of systems (1) and (4) can be realized under the observer (5) and the controller (6) constructed by Algorithm 3.

Algorithm 3. 1. Solve the following LMI:

$$
\left(\begin{array}{cc}
P A+A^{T} P-\sigma C^{T} C+\varepsilon_{5} l^{2} I & P D \\
* & -\varepsilon_{5} I
\end{array}\right)<0
$$

to get a matrix $P>0$ and positive scalars $\varepsilon_{5}$ and $\sigma$.

2. Solve the following LMI:

$$
\left(\begin{array}{ccc}
A Q+Q A^{T}-\kappa B B^{T} & D & Q \\
* & -\varepsilon_{6} I & 0 \\
* & * & -\varepsilon_{6}^{-1} l^{-2} I
\end{array}\right)<0
$$

to get a matrix $Q>0$ and positive scalars $\varepsilon_{6}$ and $\kappa$. The rest of variables, the form of the observer and the controller are the same as those in Algorithm 1.

Corollary 3. Under Assumptions 3 and 4, if inequality (40) holds and the LMIs (41-44) have feasible solutions, the consensus tracking of systems (1) and (4) can be reached exponentially fast under the observer-type protocol (20-a, b) constructed by Algorithm 4.

$$
\delta \cdot \sigma_{k}>\eta \cdot \rho_{k}+\left(h_{k}-1\right) \cdot \ln r_{0} .
$$

where positive parameters $\delta$ and $\eta$ are determined from (40), and then substituted into the LMIs (41-44).

Algorithm 4. 1. Solve the following LMI:

$$
\begin{gathered}
\left(\begin{array}{cc}
P A+A^{T} P-\sigma C^{T} C+\varepsilon_{7} l^{2} I+\delta P & P D \\
* & -\varepsilon_{7} I
\end{array}\right)<0 \\
\left(\begin{array}{cc}
P A+A^{T} P-\sigma C^{T} C+\varepsilon_{7} I^{2} I-\eta P & P D \\
* & -\varepsilon_{7} I
\end{array}\right)<0
\end{gathered}
$$

to get a matrix $P>0$ and positive scalars $\varepsilon_{7}$ and $\sigma$.

2. Solve the following LMI:

$$
\left(\begin{array}{ccc}
A Q+Q A^{T}-\kappa B B^{T}+\delta Q & D & Q \\
* & -\varepsilon_{8} I & 0 \\
* & * & -\varepsilon_{8}^{-1} l^{-2} I
\end{array}\right)<0
$$




$$
\left(\begin{array}{ccc}
A Q+Q A^{T}-\kappa B B^{T}-\eta Q & D & Q \\
* & -\varepsilon_{8} I & 0 \\
* & * & -\varepsilon_{8}^{-1} l^{-2} I
\end{array}\right)<0
$$

to get a matrix $P>0$ and positive scalars $\varepsilon_{8}$ and $\sigma$. The rest of variables, the form of the observer and the controller are the same as those in Algorithm 2.

Remark 5. The state feedback protocols were proposed in [2,3] for achieving consensus of multi-agent systems with Lipschitz, and corollary 1 extends it to the observer-based protocol. Furthermore, Corollary 2 also extends the continuous controller by outputs feedback [19] to the intermittent case. Therefore, the results given here are more general than the existing ones.

On the other hand, the two variables $\varepsilon_{5}$ and $\varepsilon_{6}$ are brought in conditions (38) and (39) to increase free degree of design. Hence, the existing result (Theorem 2 in literature [19]) can be recovered from our result, which is elaborated as follows.

By letting $\varepsilon_{5}=l^{-2}$, the LMI (38) is Schur equivalent to:

$$
P A+A^{T} P-\sigma C^{T} C+I+l^{2} P D D^{T} P<0 .
$$

But the LMI (27) in [19] is Schur equivalent to:

$$
P A+A^{T} P-\sigma C^{T} C+I+l^{2}\|D\|^{2} P P<0 .
$$

It is easy to get that the left side of the LMI (45) is no greater than that of the LMI (46). By letting $\varepsilon_{6}=l^{-2}$, the LMI (39) is Schur equivalent to the LMI (28) in literature [19].

\section{Simulation examples}

In this section, two simulation examples are provided to verify the effectiveness of Theorems 1 and 2, respectively.

\section{Example 1. Mechanical revolving model}

Fig. 2 shows the schematic of a physical system [39]. In the figure, $J$ represents inertia of the revolving cylinder; $R$, the radius of the cylinder; $H$, the viscous friction coefficient of the revolving part; $\theta$, the angular rotation, $F$, the force added by a motor whose power is a constant value, while $\Delta F$ is input force from the time-varying power.

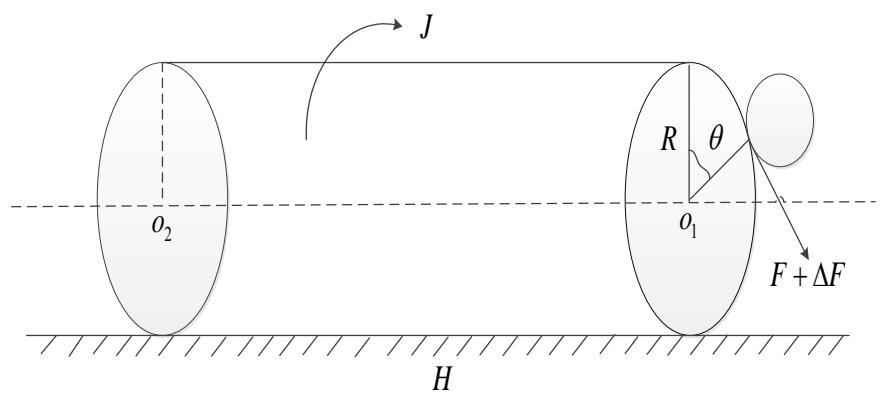

Fig. 2. A mechanical revolving model. 
A state space description of this system is given by:

$$
\left\{\begin{array}{l}
\dot{\theta}=\omega \\
\dot{\omega}=-\frac{H}{J} \omega+\frac{a}{J \omega}+\frac{\Delta F R}{J}
\end{array}\right.
$$

where $\omega$ is the angular velocity, $F R \omega=a, a$ is a constant, i.e. $F=\frac{a}{R \omega}$. The system can be described by the (1) with appropriate parameter, where $A=\left(\begin{array}{cc}0 & 1 \\ 0 & -1.2\end{array}\right), B=\left(\begin{array}{l}0 \\ 1\end{array}\right)$, $C=\left(\begin{array}{ll}1 & 0\end{array}\right), \quad D=\left(\begin{array}{ll}1 & 0 \\ 0 & 1\end{array}\right), \Phi(x, t)=\left(\begin{array}{c}0 \\ \frac{4.25}{x_{2}}\end{array}\right), x=\left(\begin{array}{c}\theta \\ \omega\end{array}\right), u=\Delta F$. Obviously, $\Phi(x, t)$ is not a Lipschitz function in $\left.D_{3}=x \in \square^{2} \mid x_{1} \in \square, x_{2}>0\right\}$. However, it is one-sided Lipschitz in $\square^{2}$ with $\rho=0$, and it is also quadratic inner bounded in $D_{2}=\left\{x \in \square^{2} \mid x_{1} \in \square, x_{2} \in[1,+\infty)\right\}$ with $\mu \geq v+1$. We can take $\mu=2$ and $v=-0.5$.

The problem is formulated as follows. Let one unforced system be the leader agent, which produces a desired state trajectory. The rest of four systems act as the follower agents, and these agents can acquire output information from their neighbors. The interaction topology of agents is described in Fig. 3. All the weights on the edges are one. The objective of consensus tracking control is to design protocol $u_{i}$ for follower agent $i$, such that their rotation angles and angular velocities can synchronize to those of the leader agent. By calculations, we have $\theta_{\max }=2$, and $\lambda_{1}=0.3290$. Taking $\varepsilon_{3}=10, \varepsilon_{4}=20$, and solving the LMIs (22-25), one has

$$
\begin{gathered}
P=\left(\begin{array}{cc}
212.4748 & 40.2710 \\
40.2710 & 335.5439
\end{array}\right), Q=\left(\begin{array}{cc}
1.9388 & -0.0002 \\
-0.0002 & 0.0468
\end{array}\right), \\
\varepsilon_{1}=248.1737, \varepsilon_{2}=478.3136, \sigma=634.3238, \kappa=516.7981 .
\end{gathered}
$$

Then, the observer and controller gain matrices are given by, respectively

$$
F=\left(\begin{array}{c}
0.0048 \\
-0.0006
\end{array}\right), K=\left(\begin{array}{ll}
0.5 & 0
\end{array}\right) \text {. }
$$

Set $\alpha=3.2 \times 10^{3} \geq \frac{\kappa \theta_{\max }}{\lambda_{1}}=3.1416 \times 10^{3}$ and. $\beta=3.9 \times 10^{3} \geq \frac{\sigma \theta_{\max }}{\lambda_{1}}=3.8561 \times 10^{3}$, The initial conditions of $s(0), x_{i}(0), \hat{x}_{i}(0)$ are randomly chosen from the square $[-4,4] \times[-4,4]$. 
As show in Fig. 4 and Fig. 5, both the estimation errors $x_{i}-\hat{x}_{i}$ and the tracking errors $X_{i}-S$ are approaching to zero. Thus, consensus tracking is achieved when the observer-protocol (5-6) is applied to the four followers.

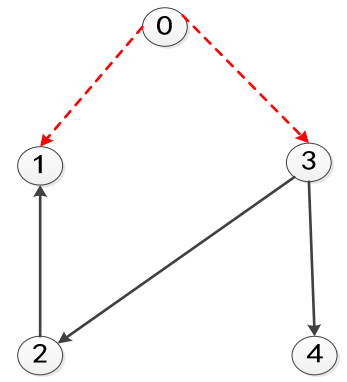

Fig. 3. Network topology $\tilde{G}^{1}$

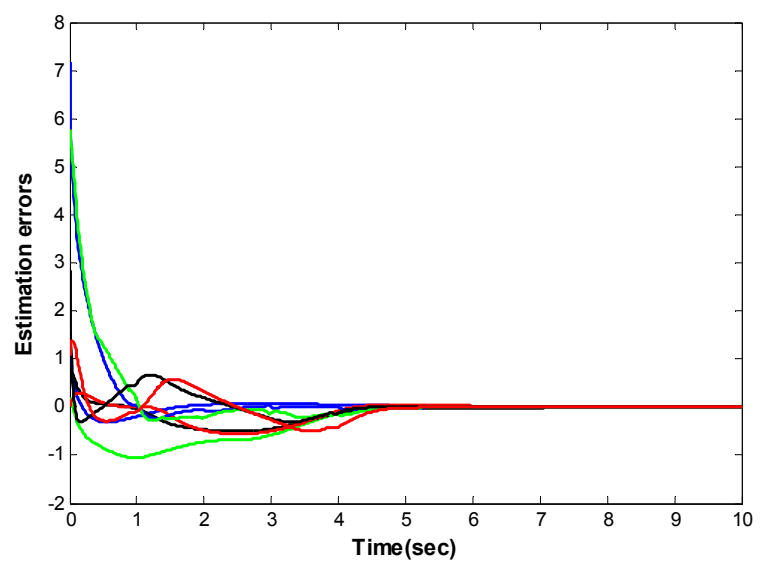

Fig. 4. Evolution of the estimation errors $x_{i}-\hat{x}_{i}$ for $i=1,2,3,4$.

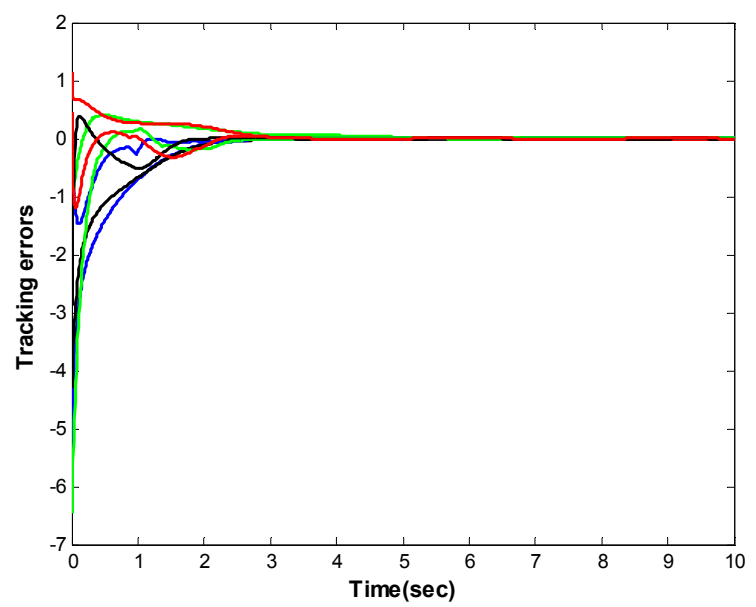

Fig. 5. Evolution of the tracking errors $x_{i}-s$ for $i=1,2,3,4$. 


\section{Example 2. Supercritical Hopf bifurcation system}

Consider a multi-agent system consisting of four followers and a leader, described respectively by (1) and (4) with

$$
A=\left(\begin{array}{cc}
\tau & -1 \\
1 & \tau
\end{array}\right), \quad D=\left(\begin{array}{ll}
1 & 0 \\
0 & 1
\end{array}\right), B=\left(\begin{array}{l}
0 \\
1
\end{array}\right), C=\left(\begin{array}{ll}
0 & 1
\end{array}\right), \quad \tau \in \square
$$

and one-sided Lipschitz fuction $\Phi\left(x_{i}, t\right)=\left(\begin{array}{c}-x_{i 1}\left(x_{i 1}^{2}+x_{i 2}^{2}\right) \\ -x_{i 2}\left(x_{i 1}^{2}+x_{i 2}^{2}\right)\end{array}\right), i=1,2,3,4$. The leader (1) is represented in the polar coordinates $s_{1}=r \cos \theta$, and $s_{2}=r \sin \theta$ by

$$
\dot{r}=\tau r-r^{3} \text { and } \dot{\theta}=1 .
$$

The system (47) describes the motion of a moving object mode, and is also a famous example about supercritical Hopf bifurcation, whose dynamics behaviors are elaborated in [40]. From [20], we know that $\Phi\left(x_{i}, t\right)$ are one-sided Lipschitz in $\square^{2}$ and $\rho=0$. Also, the system is locally Lipschitz in $D_{3}=\left\{x \in \square^{2}:\|x\|<\tilde{r}\right\}$, the Lipschitz constant $l$ is $3 \tilde{r}^{3}$, i.e., the Lipschitz constant rapidly increases with the increase of $\tilde{r}$. Letting $D_{2}=\left\{x \in \square^{2}:\|x\|<\bar{r}\right\}$, some calculations yield $\bar{r}=\min \left(\sqrt{-\frac{v}{4}}, \sqrt[4]{\mu+\frac{v^{2}}{4}}\right)$, and thus $\Phi\left(x_{i}, t\right)$ are also the quadratic inner bounded, when $v<0, \mu+\frac{v^{2}}{4}>0$ [16]. We take $u=0.01, v=-0.01, \tau=1$. The switching topologies are illustrated in Fig. 6, where the graph is $\tilde{G}^{\sigma(t)}=\tilde{G}^{1}$ for $t \in[k, k+0.4) \quad \mathrm{s}, \quad \tilde{\mathrm{G}}^{\sigma(t)}=\tilde{\mathrm{G}}^{2} \quad$ for $\quad t \in[k+0.4, k+0.9) \quad \mathrm{s}, \quad$ and $\quad \tilde{\mathrm{G}}^{\sigma(t)}=\tilde{\mathrm{G}}^{0} \quad$ for $t \in[k+0.9, k+1)$ s. All the weights on the edges are one. By calculations, we get that $r_{1}=2$, $r_{0}=2$ and $\lambda_{2}=0.5273$. From (30), we can take $\eta=15$ and $\delta=4$. By taking $\varepsilon_{3}=10, \varepsilon_{4}=20$ and solving the LMIs (22-25), we obtain

$$
\begin{gathered}
P=\left(\begin{array}{cc}
3.3771 & -13.0087 \\
-13.0087 & 84.5619
\end{array}\right), Q=\left(\begin{array}{ll}
0.0664 & 0.3055 \\
0.3055 & 3.1408
\end{array}\right), \\
\varepsilon_{1}=2.9969, \quad \varepsilon_{2}=20.9288, \sigma=7.7429, \quad \kappa=2.5348 .
\end{gathered}
$$

Then, the observer and controller gain matrices are given by, respectively 


$$
F=\left(\begin{array}{l}
0.1118 \\
0.0290
\end{array}\right), K=\left(\begin{array}{ll}
0 & 0.5
\end{array}\right)
$$

Set $\alpha=10>\frac{\kappa \cdot r_{1}}{\lambda_{2}}=9.6143$ and $\beta=30>\frac{\sigma \cdot r_{1}}{\lambda_{2}}=29.3681$. The initial conditions of $s(0), x_{i}(0), \hat{x}_{i}(0)$ are randomly chosen from the square $[-4,4] \times[-4,4]$. As shown in Fig. 7 and Fig. 8, both the estimation errors $x_{i}-\hat{x}_{i}$ and the tracking errors $x_{i}-s$ are approaching to zero. Thus, consensus tracking is achieved when the intermittent protocol (24-a, b) is applied to the four followers.

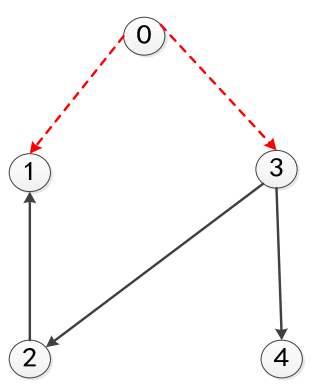

(a) $\tilde{G}^{1}$.

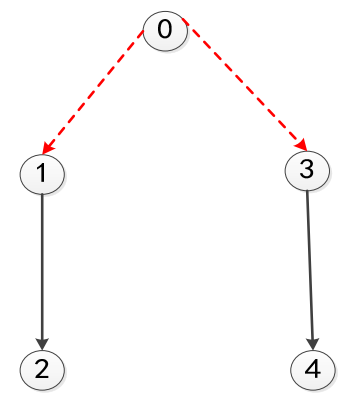

(b) $\tilde{G}^{2}$
0

(1)

(c) $\tilde{G}^{0}$

Fig. 6. Switching networks. 


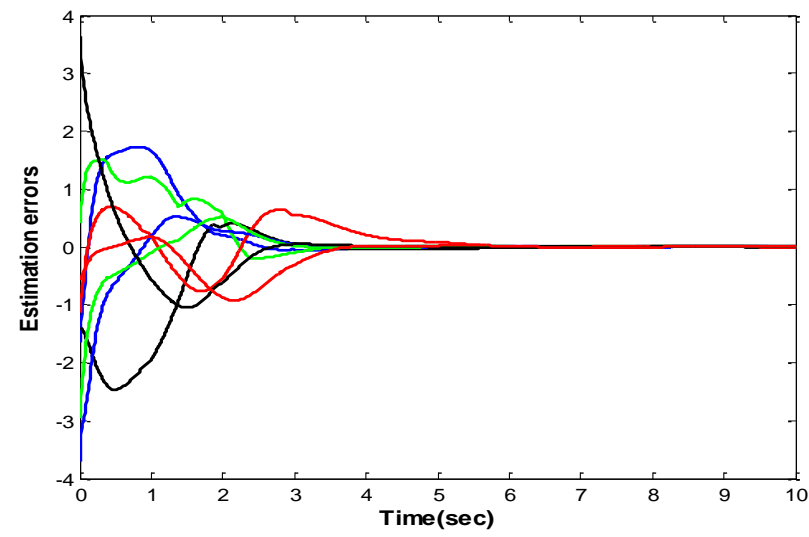

Fig. 7. Evolution of the estimation errors $x_{i}-\hat{x}_{i}$ for $i=1,2,3,4$.

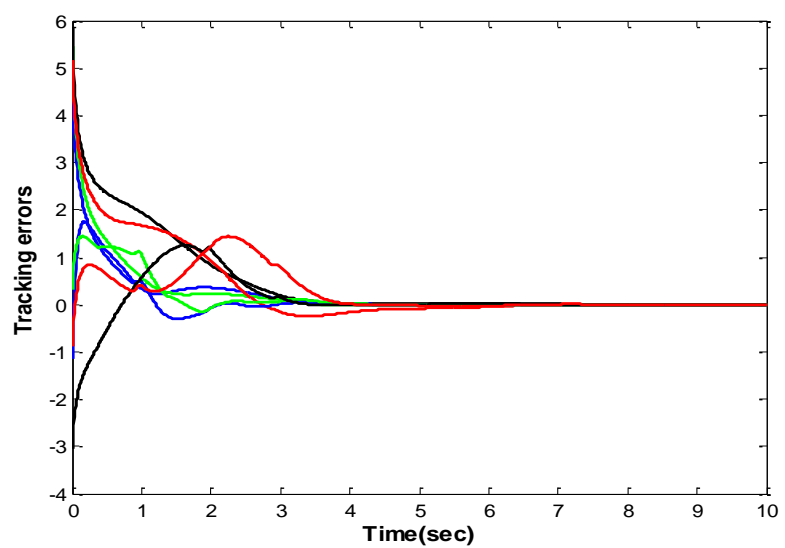

Fig. 8. Evolution of the tracking errors $x_{i}-s$ for $i=1,2,3,4$.

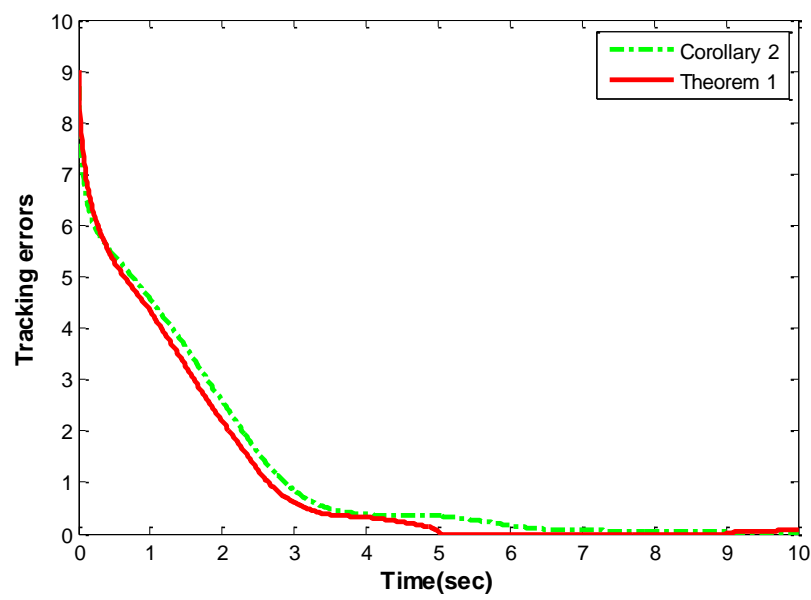

Fig. 9. Evolution of $\left\|e_{2}\right\|$ 


\section{Conclusion}

This paper has addressed the consensus tracking problem for general linear multi-agent systems with one-sided Lipschitz nonlinearity. Two types of observer-based protocols have been designed, under which it has been theoretically shown that both the estimation errors and the tracking errors approach to zero. The obtained results are more general

and less conservative than the existing ones. Finally, two numerical examples are given to illustrate the effectiveness of the proposed design approach. Future work is to consider the cooperative control problems for other types of nonlinear multi-agent systems.

\section{Acknowledgement}

This paper is partly supported by the National Science Foundation of China (61025016, 61473183, 61034008, 61221003, 61374160), Program of Shanghai Subject Chief Scientist (14XD1402400), and SJTU M\&E Joint Research Foundation (YG2013MS04).

\section{References}

[1] Y. Cao, W. Yu, W. Ren, and G. Chen. An overview of recent progress in the study of distributed multi-agent coordination. IEEE Transactions on Industrial Informatics, 2013, 9(1): 427-4384.

[2] G. Wen, Z. Duan and G. Chen. Consensus tracking of multi-agent systems with Lipschitzt type node dynamics and switching topologies. IEEE Transactions on Circuits and Systems I: Regular Papers, 2014, 61(2): 499-511.

[3] Z. Li, and D. Liu. Global $H_{\infty}$ consensus of multi-agent systems with Lipschitzt non-linear dynamics. IET Control Theory and applications, 2012, 6(13): 2041-2048.

[4] M. Stuart and A. R. Humphries. Dynamical systems and numerical analysis. Cambridge University Press, 1998

[5] K. Dekker and J. G. Verwer. Stability of Runge-Kutta methods for stiff nonlinear differential equations. North-Holland, 1984.

[6] E. Hairer, S. P. Norsett and G. Wanner. Solving ordinary differential equations II: stiff and DAE problems. Springer-Verlag, 1993

[7] M. Moisan, O. Bernard and J. Gouz. Near optimal interval observers bundle for uncertain bioreactors. Automatica, 2009, 45(1): 291-301

[8] M. Abbaszadeh and H. Marquez. Nonlinear observer design for one-sided Lipschitz systems, Proceedings of the 2010 American Control Conference, 2010, 5284-5289.

[9] M. Benallouch, M. Boutayeb and M. Zasadzinski. Observer design for one-sided Lipschitz discrete-time systems. Systems \& Control Letters, 2012, 61(9): 879-886.

[10] S. E. Tuna, Synchronizing linear systems via partial-state coupling. Automatica, 2008, 44(8): 2179-2184.

[11] B. Zhou, C. Xu and G. Duan. Distributed and truncated reduced-order observer based output feedback consensus of multi-agent systems. IEEE Transactions on Automatic Control, 2014, 59(8): 2264-2270.

[12] Y. Hong, J. Hu, and L. Gao. Tracking control for multi-agent consensus with an active 
leader and variable topology. Automatica, 2006, 42(7): 1177-1182.

[13] J. Mei, W. Ren and G. Ma. Distributed coordination for second-order multi-agent systems with nonlinear dynamics using only relative position measurements. Automatica, 2013, 49(5): 1419-1427.

[14] X. Lu, R. Lu, S. Chen, and J. Lü. Finite-time distributed tracking control for multi- agent systems with a virtual leader. IEEE Transactions on Circuits and Systems I: Regular Papers, 2013, 60(2): 352-362.

[15] Z. Li, Z. Duan, G. Chen, and L. Huang. Consensus of multiagent systems and synchronization of complex networks: a unified viewpoin. IEEE Transactions on Circuits and Systems I: Regular Papers, 2010, 57(1): 2213 - 2242.

[16] Z. Li, X. Liu, P. Lin, and W. Ren. Consensus of linear multi-agent systems with reduced-order observer-based protocols. Systems \& Control Letters, 2011, 60(7): 510-516.

[17] H. Zhang, F. L. Lewis, and A. Das. Optimal design for synchronization of cooperative systems: state feedback, observer, and output feedback. IEEE Transactions on Automatic Control, 2013, 56(8): 1948-1952.

[18] H. M. Kristian and F. L. Lewis. Cooperative observers and regulators for discrete-time multi-agent systems. International Journal of Robust and Nonlinear Control, 2013, 23(14): $1545-1562$

[19] J. Hu and J. Cao. Consensus of nonlinear multi-agent systems with observer-type protocols. System control letter. 2014, 72(10): 71-79.

[20] W. Zhang, H. Su, Y. Liang and Z. Han. Non-linear observer design for one-sided Lipschitz systems: an linear matrix inequality approach. IET Control Theory and applications, 2012, 6(9): 1297-1303.

[21] W. Zhang, H. Su, F. Zhu and D. Yue. A note on observers for discrete-time Lipschitz nonlinear systems. IEEE Transactions on Circuits and Systems II: Express Brief. 2012, 59(2): 123-127.

[22] W. Zhang, H. Su, H. Wang and Z. Han. Full-order and reduced-order observers for one-sided Lipschitz nonlinear systems using Riccati equations. Commun Nonlinear Sci Numer Simulat, 2012, 17(12): 4968-4977.

[23] M. Chen and C. Chen. Robust nonlinear observer for Lipschitz nonlinear systems subject to disturbances. IEEE Trans Automat Control, 2007, 52(12): 2365-2369.

[24] M. Zochowski. Intermittent dynamical control. Physica D, 2000, 145 (3-4): 181-190.

[25] G. Wen, G. Hu, W. Yuan, J. Cao and G. Chen. Consensus tracking for higher-order multi-agent systems with switching directed topologies and occasionally missing control inputs. Systems \& Control Letters, 2013, 62(12): 1151-1158.

[26] H. Su, G. Jia and M. Z. Q. Chen. Semi-global containment control of multi-agent systems with intermittent input saturation. Journal of the Franklin Institute, 2015, 352(9): 3504-3525.

[27] Y. Tang and W. K. Wong. Distributed synchronization of coupled neural networks via randomly occurring control. IEEE Transactions on Neural Networks and Learning Systems, 2013, 24(3): 435-446.

[28] H. Zhang and F. L. Lewis. Adaptive cooperative tracking control of high-order nonlinear systems with unknown dynamics. Automatica, 2010, 48(7): 1432-1439. 
[29] F. Zhang, H. L. Trentelman and M. A. Jacquelien. Fully distributed robust synchronization of networked Lur'e systems with incrental nonliearities. Automatica, 2014, 50 (2): 515-525.

[30] P. DeLellis and M. D. Bernardo. On QUAD, Lipschitz, and contracting vector fields for consensus and synchronization of networks. IEEE Transactions on Circuits and Systems I: Regular Papers, 2011, 58 (3): 576-583.

[31] W. Lu, X. Li and Z. Rong. Global stabilization of complex networks with digraph topologies via a local pinning algorithm. Automatica, 2010, 46 (1): 116-121.

[32] Y. Han, W. Lu, Z. Li and T. Chen. Pinning dynamic systems of networks with Markov switching couplings and controller-node set. Systems \& Control Letters, 2014, 65(2): 56-63.

[33] W. Ni and D. Cheng. Leader-following consensus of multi-agent systems under fixed and switching topologies. Systems \& Control Letters, 2010, 59(3): 209-217.

[34] H. Su, M. Z. Q. Chen and X. Wang. Semiglobal observer-based leaderfollowing consensus with input saturation. IEEE Transactions on Industrial Electronics, 2014, 61(6): 2842-2850.

[35] H. Su, M. Z. Q. Chen, J. Lam and Z. Lin. Semi-global leader-following consensus of linear multi-agent systems with input saturation via low gain feedback. IEEE Transactions on Circuits and Systems I: Regular Papers, 2013, 60(7): 1881-1889.

[36] D. Liberzon. Switching in systems and control. Berlin: Birkhauser (2003).

[37] H. Yang, B. Jiang and J. Zhao. On finite- time stability of cyclic switched nonlinear systems. IEEE Transactions on Automatic Control, 2015, 60(8): 2201-2206.

[38] H. Yang, B. Jiang, V. Cocquempot and H. Zhang. Stabilization of switched nonlinear systems with all unstable modes: applications to multi-agent systems, IEEE Transactions on Automatic Control, 2011, 56(9), 2230-2235.

[39] M. Xu, G. Hu, Y. Zhao. Reduced-order observer design for one-sided Lipschitz non-linear systems. IMA Journal of Mathematical Control and Information. 2009, doi:10.1093/imamci/dnp017.

[40] K. Hassan. Nonlinear systems (Third edition). Prentice Hall, 2002. 